\title{
GESTÃO DE RESÍDUOS EM LABORATÓRIOS CLÍNICOS DA REDE PRIVADA DE PRESIDENTE PRUDENTE/SP: ANÁLISE DA LOGÍSTICA REVERSA
}

\author{
Jéssica Ramos Magalhães Olegário ${ }^{1}$ \\ Lechan Colares-Santos $^{2}$
}

Sueli Cristina Schadeck Zago ${ }^{3}$

\begin{abstract}
Resumo
A área da saúde vem sofrendo mudanças no que diz respeito à sua legislação, vários laboratórios tiveram que se adequar as novas normas criadas pelo governo principalmente no que diz respeito à gestão dos resíduos, onde a responsabilidade recai sobre o gerador. A preocupação com o gerenciamento correto dos Resíduos Sólidos - RS deu-se através da aprovação da Lei Federal no 12.305/2010, que obriga e delega a responsabilidade ao gerador em destinar corretamente os resíduos por ele gerado. Neste contexto, a Logística Reversa é uma ferramenta determinante no gerenciamento dos resíduos, pois é responsável pelo planejamento, operação e controle do fluxo de resíduos gerados, possibilitando que os mesmos sejam descartados de maneira ecologicamente correta. O objetivo desta pesquisa foi identificar e analisar as práticas adotadas pelos laboratórios clínicos privados da cidade de Presidente Prudente/SP no tratamento dos resíduos por eles gerados. A pesquisa foi desenvolvida de forma descritiva, exploratória, qualitativa, com aplicação de estudo multicaso, utilizando a técnica análise de conteúdo, tendo como ferramenta de coleta de dados a realização de entrevistas face a face. O presente estudo identificou que os laboratórios adotam práticas adequadas de gestão dos resíduos. Mediante os resultados apresentados, observou-se que os laboratórios de análises clínicas privados de Presidente Prudente, atendem às normas das agências reguladoras, tratando os seus resíduos conforme as normas exigidas na literatura específica. No entanto, esse comportamento está exclusivamente associado ao atendimento da legislação, tendo como fator secundário a segurança ambiental.
\end{abstract}

\footnotetext{
${ }^{1}$ Graduanda em Administração, Universidade do Oeste Paulista (UNOESTE). jessicaramosmagalhaes@hotmail.com.

${ }^{2}$ Mestre em Administração, prof.. do curso de Administração da Universidade do Oeste Paulista (UNOESTE). lechan@unoeste.br.

3Mestre em Farmácia e Bioquímica, prof.ำ do curso de Medicina, Odontologia, Nutrição, Farmácia e Enfermagem da Universidade do Oeste Paulista (UNOESTE). sueli@unoeste.br.
} 
Palavras-chave: Logística Reversa. Laboratório de Análises Clínicas. Resíduos de Serviços de Saúde.

\title{
WASTE MANAGEMENT IN CLINICAL LABORATORIES OF PRIVATE NETWORK OF PRESIDENTE PRUDENTE/SP: ANALYSIS OF THE REVERSE LOGISTICS
}

\begin{abstract}
The health sector has changed as regards its legislation, several laboratories had to fit the new standards set out by the government especially in the issue of waste management, where the onus is on the generator. Concern for the proper management of solid waste - RS occurred by adopting Federal Law No. 12,305 / 2010, which obliges and delegates responsibility to the generator to properly allocate the waste they generate. In this context the Reverse Logistics is a crucial tool in the management of waste, it is responsible for planning, operation and control of the flow of waste generated, enabling them to be disposed of in an environmentally sound manner. The objective of this research was to identify and analyze the practices adopted by individual clinical laboratories in the city of Presidente Prudente / SP in the treatment of waste generated by them. The research was conducted in a descriptive, exploratory, qualitative way with multi case study application using the content analysis technique, with the data collection tool to carry out face to face interviews. This study identified that laboratories adopt appropriate waste management practices. From the results presented it was observed that private clinical laboratories of Presidente Prudente / SP meet the standards of regulatory agencies, treating their waste according to the standards required in the literature, however, this behavior is exclusively associated with compliance with legislation, having as a secondary factor environmental safety.
\end{abstract}

Keywords: Reverse Logistics. Clinical Analysis Laboratory. Health Services waste.

\section{GESTIÓN DE RESIDUOS EN LOS LABORATORIOS CLÍNICOS DE LA RED PRIVADA DE PRESIDENTE PRUDENTE/SP: ANÁLISIS DE LA LOGÍSTICA INVERSA}

\begin{abstract}
Resumen
El sector de la salud ha cambiado en cuanto a su legislación, varios laboratorios tuvieron que adaptarse a las nuevas normas establecidas por el gobierno, especialmente en el tema de la gestión de residuos, donde la responsabilidad recae en el generador. La preocupación por el manejo adecuado de residuos sólidos - RS se produjo mediante la adopción de la Ley Federal № 12.305 / 2010, que obliga y delega la responsabilidad al generador para asignar adecuadamente los residuos que generan. En este contexto, la logística inversa es una herramienta fundamental en la gestión de los residuos, es responsable de la planificación, operación y control del flujo de los residuos
\end{abstract}


generados, lo que les permite ser eliminados de una manera ambientalmente racional. El objetivo de esta investigación fue identificar y analizar las prácticas adoptadas por los laboratorios clínicos individuales en la ciudad de Presidente Prudente / SP en el tratamiento de los residuos generados por los mismos. La investigación se llevó a cabo de una manera descriptiva, exploratoria, cualitativa con la aplicación del estudio de varios casos utilizando la técnica de análisis de contenido, con la herramienta de recolección de datos para llevar a cabo entrevistas cara a cara. Este estudio identificó que los laboratorios adopten prácticas adecuadas de gestión de residuos. A partir de los resultados presentados se observó que los laboratorios clínicos privados de Presidente Prudente / SP cumplen las normas de los organismos reguladores, el tratamiento de sus residuos de acuerdo a los estándares requeridos en la literatura, sin embargo, este comportamiento se asocia exclusivamente con el cumplimiento de la legislación, teniendo como un factor secundario seguridad ambiental.

Palabras clave: logística inversa. Laboratorio de Análisis Clínicos. Residuos Servicios de Salud.

\section{INTRODUÇÃO}

A incessante preocupação com a busca pela qualidade de vida procede, essencialmente, da procura por um ambiente equilibrado e saudável. Os serviços relacionados à saúde são indispensáveis para atingir qualidade e bom desenvolvimento da vida humana. Assim, a necessidade deste tipo de serviço aumentou significativamente nos últimos anos, contribuindo para o aumento da produção de resíduos gerados na prestação dos mesmos.

No início do século XXI um dos principais problemas enfrentados pela sociedade é a crescente geração de Resíduos de Serviços de Saúde - RSS. Os RSS gerados nas unidades de saúde, tanto pública quanto privada, compõem um dos atuais problemas relacionados à saúde pública e ambiental, pois são resíduos que necessitam de técnicas e cuidados especiais em seu gerenciamento por conter características infecciosas e contaminantes. Relacionando-se o crescente volume de resíduos gerados com a falta do correto gerenciamento por parte dos responsáveis, observa-se que muitos resíduos possuem destinação final inadequada, fato que compromete a saúde como um todo na sociedade e no meio ambiente, contribuindo, inclusive e consequentemente, para o aumento de problemas econômicos. 
Como não existe a possibilidade de deixar de gerar resíduos durante esses procedimentos clínicos, é fundamental buscar alternativas que possam controlar os danos ocasionados por esses resíduos. Uma das formas encontradas para minimizá-los, é a adoção da Logística Reversa, uma ferramenta de gestão utilizada para destinar os resíduos conforme sua classificação, para um local específico, sem acarretar problemas sócio-ambientais e econômicos. Desta maneira, torna-se importante identificar e analisar as práticas adotadas pelos laboratórios de análises clínicas no tratamento dos resíduos por eles gerados.

Diante disto, esta pesquisa é de significativa importância, pois contribui para o avanço dos estudos relacionados ao gerenciamento de resíduos de serviços de saúde, procurar uma relação entre as variáveis ainda não exploradas para os laboratórios de análises clínicas pesquisados, contribui como diagnóstico, subsidiando decisões de gestores, proporcionando melhorias no bem estar da sociedade analisada. Para tanto, formulou-se a seguinte questão de pesquisa: como se dão as práticas de logística reversa adotadas pelos laboratórios clínicos privados na cidade de Presidente Prudente?

Com o intuito de responder a esta questão, o presente estudo tem por objetivo identificar e analisar as práticas adotadas pelos laboratórios clínicos privados da cidade de Presidente Prudente/SP no tratamento dos resíduos por eles gerados.

\section{Resíduos Hospitalares}

"Os resíduos sólidos dos serviços de saúde (RSS) são a nomenclatura mais recente correspondente aos "resíduos hospitalares", denominação antiga e hoje inadequada, embora de uso consagrado" (BARROS, 2012, p.339). Deste modo, os resíduos hospitalares passam a serem chamados resíduos de serviços de saúde, os resíduos de serviços de saúde correspondem a cerca de $1 \%$ a $3 \%$ do total de resíduos urbanos gerados. A importância dispendida com esses resíduos não está 
associada somente a quantidade gerada, mas, o potencial de risco que representam a saúde ambiental e social (ANVISA, 2006).

"Os resíduos de serviço de saúde são constituídos de todo e qualquer resíduo gerado dentro de uma unidade de saúde, sendo estes classificados de acordo com o tipo e o grau de contaminação" (PEREIRA, 2012, p. 91). Sendo assim, grande parte dos resíduos é oriundo exclusivamente de unidades prestadoras de serviços de saúde, entretanto, podem também ser gerados em residências e em locais de trabalho, contribuindo para o aumento do grau de contaminação, uma vez que as informações sobre o manuseio ainda são escassas. No entanto, "a falta de informações sobre o assunto é um dos principais motivos para a ausência de projetos bem sustentados que determinam melhorias no setor" (NAIME, SARTOR e GARCIA, 2004, p. 18). Desta forma, a questão central não está voltada somente para a geração ou a minimização da quantidade de resíduos gerada, mas sim para o tratamento adequado, visando obter melhorias no setor da saúde com o propósito de reduzir ou até mesmo extinguir os passivos negativos por eles gerados.

Para o desenvolvimento de projetos que visem avaliar os resíduos de serviços de saúde - RSS é necessário conhecer os diversos tipos de resíduos gerados, quais as suas características e, o tratamento correto pelo qual devem ser submetidos, pois somente assim, será possível elaborar estratégias que visem proporcionar segurança durante o gerenciamento e, desta forma, não importa o tamanho do estabelecimento de saúde, todos deverão elaborar um Plano de Gerenciamento de Resíduos de Serviços de Saúde - PGRSS, adequando-se às normas, para o pleno funcionamento e gerenciamento correto dos resíduos por eles gerados.

Segundo a Lei Estadual n 12.300 de 16 de março de 2006, Art. 14 - IX é proibido o encaminhamento de resíduos de serviços de saúde para disposição final em aterros, sem submetê-los previamente a tratamento específico, que neutralize sua periculosidade. Desta maneira, os RSS são resíduos que precisam de técnicas especiais em seu tratamento, além disso, é necessária a realização de etapas como 
a de identificação e classificação dos RSS, pois, apenas mediante o cumprimento destas é que esses resíduos poderão ser submetidos a uma destinação final correta, a fim de minimizar ou neutralizar os impactos por eles gerados.

A identificação e classificação dos grupos de resíduos existentes, proporcionam a realização correta do gerenciamento dos RSS, contribuindo para a elaboração de estratégias voltadas ao manejo, segregação, acondicionamento e descarte dos mesmos, minimizando os riscos de contaminação dos profissionais que atuam nesta área, o que, inclusive, minimiza os passivos negativos advindos do mau gerenciamento, geradores de problemas que afetam a sociedade e o meio ambiente, causando danos, muitas vezes, irreversíveis. A identificação contribui para melhorias nas estratégias já estabelecidas acerca das etapas de gerenciamento e, coopera para o desenvolvimento de novas estratégias voltadas ao equilíbrio ambiental e a sustentabilidade além de desenvolver normas que visam à segurança para a saúde pública, contribuindo para o apoio de uma gestão adequada.

Os RSS são classificados em cinco grupos: o grupo A caracterizado por resíduos biológicos; grupo B caracterizado por resíduos químicos; grupo C caracterizado por resíduos radioativos; grupo $D$ caracterizado por resíduos comuns; grupo E caracterizado por perfurocortantes ou escarificantes (ANVISA, 2006). Sendo assim, esses resíduos necessitam de cuidados ou até mesmo técnicas especiais em seu gerenciamento, pois possuem alto risco de contaminação, se manuseados, segregados, acondicionados, transportados e descartados de maneira inadequada.

Os estabelecimentos de serviços de saúde são os responsáveis pelo correto gerenciamento de todos os RSS por eles gerados, cabendo aos órgãos públicos, dentro de suas competências, a gestão, regulamentação e fiscalização (ANVISA, 2006, p.38).

Desta forma, os estabelecimentos de saúde são os responsáveis diretos pela destinação correta dos resíduos por serem os próprios geradores, cabendo aos órgãos competentes cumprir com a fiscalização e a punição de eventuais 
estabelecimentos que estejam atuando de maneira inadequada no descarte dos RSS.

O próximo item deste estudo trata de maneira pormenorizada da Logística Reversa, uma ferramenta essencial no manuseio dos resíduos.

\section{Logística Reversa}

A Logística Reversa vem ocupando um espaço muito importante nas empresas, embora seja um tema incipiente, tem uma forte tendência de ampliar-se. Para o processo de implantação desta ferramenta é necessário investimento em capital humano e financeiro, pois são múltiplos os fatores que podem influenciar a adoção da Logística Reversa, tais como: a sustentabilidade; pressões legais que envolvem a nova legislação; reaproveitamento dos materiais; imagem diferenciada da empresa; redução de custos na fabricação e a consciência ambiental dos consumidores.

Um planejamento responsivo da LR apresenta os alicerces para implantar, operar e gerir de maneira adequada os diversos resíduos existentes, além de tornar mínimos os impactos causados à saúde do ser humano e ao meio ambiente, reduzindo o volume de resíduos espalhados e destinados de maneira incorreta no meio ambiente.

Segundo a Lei oㅜ 12.305, de 2 de agosto de 2010, que instituiu a PNRS Política Nacional de Resíduos Sólidos, conceitua-se logística reversa no Inciso XII e Art. $3^{\circ}$ como:

[...] instrumento de desenvolvimento econômico e social caracterizado por um conjunto de ações, procedimentos e meios destinados a viabilizar a coleta e a restituição dos resíduos sólidos ao setor empresarial, para reaproveitamento, em seu ciclo ou em outros ciclos produtivos, ou outra destinação final ambientalmente adequada; 
Sendo assim, a LR é uma área da logística especializada em viabilizar meios de coleta adequada dos resíduos, além de proporcionar o descarte correto e, quando o resíduo não é tido como perigoso à saúde do homem e ao meio ambiente, proporciona o reaproveitamento do mesmo. No entanto, a implantação da LR exige atividades estratégicas básicas para obter resultados significativos, que podem fazer diferença no momento da apuração dos resultados, pois, faz-se necessário o conhecimento sobre o que a organização implantará para poder gerir-se de maneira adequada, desenvolvendo de maneira satisfatória e efetiva um diferencial competitivo. Shibao, Moori, Santos (2010) apresentam cinco atividades básicas da Logística Reversa, como pode ser observado no Quadro 1.

\section{QUADRO 1 - As cinco atividades básicas da Logística Reversa}

Planejamento, implantação e controle do fluxo de materiais e do fluxo de informações do ponto de consumo ao ponto de origem;

Movimentação de produtos na cadeia produtiva, na direção do consumidor para o produtor;

Busca de uma melhor utilização de recursos, seja reduzindo o consumo de energia, seja diminuindo a quantidade de materiais empregada, seja reaproveitando, reutilizando ou reciclando resíduos;

Recuperação de valor e

Segurança na destinação após utilização.

Fonte: Shibao, Moori, Santos, (2010, p. 04).

Desta maneira, é de significativa importância realizar um planejamento, antes de implantar os processos logísticos reversos no ambiente organizacional para obter-se o pleno controle das atividades desenvolvidas e buscar a segurança na destinação dos resíduos através da responsabilidade compartilhada.

De acordo com a Lei ํo 12.305, de 2 de agosto de 2010, que instituiu a PNRS no Art. 30: 
É instituída a responsabilidade compartilhada pelo ciclo de vida dos produtos, a ser implementada de forma individualizada e encadeada, abrangendo os fabricantes, importadores, distribuidores e comerciantes, os consumidores e os titulares dos serviços públicos de limpeza urbana e de manejo de resíduos sólidos, consoante as atribuições e procedimentos previstos nesta Seção.

Ao se tratar do ciclo de vida do produto pode-se confirmar que não termina na entrega ao consumidor final, outro fator importante a ressaltar é que o ciclo de vida dos produtos tem sido reduzido, devido a novos produtos introduzidos no mercado a todo o momento, o que tem gerado aumento na produção de resíduos.

Assim, a importância da LR torna-se nítida, proporcionando assim um destino correto, seja por intermédio do reaproveitamento/reciclagem ou pela destinação correta, através dos canais reversos, contribuindo para a sustentabilidade. "Os canais de distribuição reversos têm sido estudados de forma crescente nos últimos anos, quando a carência de informações e sistematização desses conhecimentos ainda era emergente" (LEITE, 2009, p. 6).

Sendo assim, a importância de estudar os canais de distribuição reversos tem contribuído para proporcionar equilíbrio social, ambiental e econômico, uma vez que este canal é responsável pelo retorno do material descartado pela sociedade, será dado a ele um devido tratamento, sua reintrodução no mercado ou o descarte de maneira correta.

\section{MÉTODOS E PROCEDIMENTOS METODOLÓGICOS}

O presente trabalho se caracteriza como uma pesquisa descritiva, exploratória e qualitativa, realizada por meio de estudo multicaso e com o uso da técnica de análise de conteúdos para organização dos dados. Para o alcance dos objetivos propostos, a pesquisa foi dividida em duas etapas. Na primeira realizou-se uma pesquisa bibliográfica por meio de estudos em livros, artigos, legislações, 
dissertações de mestrado e teses de doutorado, além de pesquisa em websites governamentais. A segunda etapa tratou de estudo empírico, e teve como instrumento de coleta de dados, entrevista pessoal com gestores de laboratórios clínicos privados da cidade de Presidente Prudente/SP.

Primeiramente, foram coletadas informações acerca da quantidade de laboratórios clínicos privados. Esta informação foi coletada junto à Prefeitura da cidade por intermédio de um requerimento, em que foi identificado um total de 12 laboratórios clínicos privados. Do total, 5 foram visitados.

A técnica de coleta de dados foi realizada por intermédio de entrevista realizada no mês de abril de 2015, com os gestores dos laboratórios. Cada entrevista durou em média uma hora e meia e foi registrada por meio de anotações realizadas em caderno. Durante as entrevistas, foram realizadas visitas no setor técnico dos laboratórios para se conhecer os processos e identificar os resíduos gerados, neste momento foram registradas as observações que contribuíram como base para análise das práticas de gestão adotadas. O estudo de dados do presente trabalho foi conduzido por técnica de análise de conteúdo, conforme orienta Bardin.

\section{ANÁLISE DOS DADOS}

Num universo de 12 (doze) laboratórios, o estudo contemplou cinco deles, ou seja, $42 \%$ dele. Os sujeitos da pesquisa foram os gestores dos laboratórios de análise clínicas que foram entrevistados e questionados sobre as práticas de gestão adotadas na destinação final dos resíduos por eles gerados. O quadro 1 traz a identificação no estudo de cada laboratório de análises clínicas e o seu respectivo responsável com o qual foi realizado a entrevista. 


QUADRO 1: Identificação de clínicas e resp
\begin{tabular}{|c|c|} 
Clínicas & Responsável \\
\hline A & 1 \\
\hline B & 2 \\
\hline C & 3 \\
\hline D & 4 \\
\hline E & 5 \\
\hline
\end{tabular}
Fonte: elaborado pelos autores

Deste modo, a análise foi realizada com base no quadro 1 para representar os laboratórios de análises clínicas e seus respectivos responsáveis.

Os gestores dos laboratórios de análise clínica foram questionados sobre os tipos de resíduos gerados nas suas atividades.

Nos laboratórios A, B e E foram identificados Resíduos do Grupo A (Biológicos), Resíduos do Grupo B (Reagentes Químicos, sólidos ou líquidos), Resíduos do Grupo D (Similar ao lixo Doméstico). No laboratório C foram identificados além dos Resíduos do grupo D (Similar ao lixo Doméstico), Resíduos do Grupo C (Radioativos). E por fim, nos laboratórios C e D foram identificados Resíduos do Grupo A (Infectantes); Resíduos do Grupo B (Reagentes Químicos); Resíduos do Grupo D (Similar ao lixo Doméstico) e Resíduos do Grupo E (Perfurocortantes).

Os laboratórios foram questionados a respeito da frequência dos descarte dos resíduos gerados, ou seja, sobre o período de armazenagem e descarte dos resíduos. A frequência de descarte dos resíduos ocorre da seguinte forma: nos laboratórios A, C, D e E os resíduos são recolhidos diariamente da área técnica, e armazenados em local especifico até a coleta que é efetuada semanalmente por uma empresa contratada para realizar a destinação final. No entanto, o responsável 1 enfatiza que "no caso dos resíduos comuns, todos os dias são retirados do laboratório pela Prudenco, os resíduos biológicos e químicos são retirados uma vez 
por semana pela empresa contratada, exceto alguns tipos de resíduos químicos (álcool, formol) quanto bem diluído pode ser desprezado na rede de esgoto, tendo que ser paga uma taxa para a Sabesp devido à realização deste descarte e quanto aos resíduos recicláveis (papel, plástico etc), são retirados pela Cooperlix, onde o laboratório possui um acordo com esta empresa."

Já no laboratório $B$, de acordo com o responsável 2 "à frequência com que os resíduos são descartados, irá depender muito do material radioativo. Todo o material radioativo deve ser acondicionado primeiro em um tanque de decaimento interno para perder/diminuir o grau de radiação, para depois ser descartado. Alguns materiais levam cerca de meses para diminuir o grau de radiação e outros levam cerca de semanas, então varia de acordo com a característica do resíduo. Portanto, os resíduos são descartados com mais frequência semanalmente, com exceção dos que levam mais tempo".

Os laboratórios foram questionados se fazem uso de políticas formais para gestão dos resíduos. O laboratório A possui um Plano de Gerenciamento de Resíduos de Serviços de Saúde formal/escrito elaborado pelos próprios responsáveis do estabelecimento de saúde. O laboratório B possui políticas que determinam os processos a partir do momento em que os resíduos são separados, acondicionados e descartados de acordo com suas classificações. O laboratório C é um laboratório acreditado e participa de programas de controle de qualidade. Segundo o gestor 3 "As políticas de qualidade e programas de acreditação que contemplamos são de gerenciamento e gestão de resíduos analisados através de indicadores de geração destes (mensal e anual)".

O laboratório D segue as políticas descritas no PGRSS, para o pleno funcionamento do laboratório frente à responsabilidade de destinar corretamente os resíduos gerados no processo de prestação do serviço de saúde a população e até mesmo para o pleno funcionamento frente à vigilância sanitária, que fiscaliza o laboratório. Já o laboratório $E$, adota políticas formais e toma como base 0 atendimento às normas da vigilância sanitária. 
Os laboratórios foram questionados a respeito da adoção de normas externas (normas de agências reguladoras, leis, e regras que regulam a atividades dos laboratórios nos níveis federal, estadual e municipal) no exercício de suas atividades.

No laboratório A, o responsável argumenta que "tudo o que é realizado pelo laboratório segue o cumprimento da lei, pois o próprio PGRSS é exigido pela ANVISA, mesmo que não se tenha como princípio, a lei nos obriga, ou seja, tudo é exercido e cumprido de acordo com o que está previsto em lei".

No laboratório B a norma externa adotada é do órgão CNEN (Conselho Nacional de Energia Nuclear), pois, o laboratório lida com materiais radioativos e, precisa seguir essas normas descritas pelo órgão regulador para o pleno funcionamento da unidade e a realização do descarte correto.

Os laboratório C e D adotam as práticas descritas no RDC 306, conforme explica o acordo com o responsável 3 "As leis que conhecemos e adotamos são a: RDC 306 da ANVISA 07/12/2004, que dispõe sobre o Regulamento Técnico para o gerenciamento de resíduos de serviços de saúde e a Resolução CONAMA $n$ ○358/2005, que dispõe sobre o tratamento e a disposição final dos resíduos dos serviços de saúde e dá outras providências. Adota por obrigações legais e por seguir um controle de qualidade" e 4 "Conhecemos e adotamos a RDC 306 de 07 de dezembro de 2004 da ANVISA, que dispõe sobre o Regulamento Técnico para o Gerenciamento de Resíduos de Serviços de Saúde. Adotamos em cumprimento da legislação". O Laboratório E não soube responder.

Os laboratórios foram questionados sobre o conhecimento a respeito do destino final dos resíduos por eles gerados e sobre a participação da organização no fluxo dos resíduos até o seu destino final.

No laboratório $A, D$ e $E$ os gestores relatam conhecerem o destino, mas não participam na gestão do fluxo reverso. O laboratório $B$ não participa e não conhece a movimentação dos resíduos até o destino final. 
O laboratório $C$ conhece e participa até o recolhimento dos resíduos realizado pela empresa contratada. De acordo com o gestor 3 "A empresa contratada precisa estar qualificada para poder exercer este tipo de atividade de coleta dos resíduos do laboratório. Esse processo de recolhimento dos resíduos realizado pela empresa, precisaria de acompanhamento, entretanto o laboratório não participa da destinação final por questões de distância. No entanto, a confiabilidade do serviço prestado é devido à emissão de laudos e certificados, comprovando que os resíduos foram descartados de acordo com a classificação. $A$ contratação desta empresa foi mediada pelo SINDHOSPRU (sindicato dos hospitais de presidente prudente), e foi realizada uma pesquisa no instituto do Paraná, para analisar se o transporte está correto".

As organizações foram questionadas sobre a realização de capacitação junto aos colaboradores que lidam diretamente com os resíduos. No laboratório A segundo o gestor 1 "é fundamental realizar a capacitação e treinamento dos colaboradores, pois está proposto pelo próprio Plano de Gerenciamento. Os colaboradores são treinados para um bom desempenho das atividades, visando à qualidade e integridade dos serviços prestados, do ambiente de trabalho e a segurança dos colaboradores, embora a preparação do material utilizado seja feito artesanalmente".

No laboratório B, de acordo com o gestor 2 "Toda unidade de saúde de medicina nuclear precisa de um supervisor de radioproteção, dentro da própria unidade. O laboratório conta com um médico nuclear, ou seja, um supervisor de radioproteção. Os treinamentos são desenvolvidos também com a colaboração de um funcionário da CIPA - Comissão Interna de Prevenção de Acidentes. Todos os colaboradores que trabalham com o manuseio dos materiais devem utilizar um colete de chumbo e um dosimetro. É fundamental que todos os dias os colaboradores utilizem o dosimetro, pois o laboratório conta com uma empresa contratada e especializada, responsável mensalmente por monitorar a dosagem de radiação que cada colaborador recebeu". 
No laboratório $\mathrm{C}$, de acordo com o gerente 3 , "Em toda questão de procedimento tem que haver treinamentos. Tal treinamento é realizado pelo próprio responsável do laboratório, já o responsável técnico e os diretores participam de congressos e cursos externos. O treinamento é realizado para se obter uma rastreabilidade nos processos, onde todos devem participar para entender a importância de gestão e descarte eficazes. Além dos treinamentos oferecidos a todos os colaboradores, eles devem usar os EPIs: roupa de manga longa, bota ou calçado fechado impermeável, luvas e óculos de proteção".

No laboratório D, segundo o responsável 4 "os colaboradores envolvidos diretamente com os RSS são submetidos à avaliação clínica abrangendo anamnese ocupacional, exame físico e os exames complementares incluindo hemograma completo e vacinação contra hepatite e tétano. Os colaboradores envolvidos diretamente com o PGRSS são capacitados na ocasião da admissão e fazem treinamento periódico para as atividades de manejo de resíduos de serviços de saúde, desde a segregação, descarte, acondicionamento, coleta, transporte, armazenamento, tratamento e disposição final dos resíduos de serviços de saúde, incluindo a responsabilidade de higiene pessoal e de materiais. A capacitação deve incluir: noções gerais sobre o ciclo de vida dos materiais, conhecimento da legislação relativa aos RSS, definição, tipo e classificação dos resíduos e potencial de risco do resíduo, sistema de gerenciamento adotado internamente no estabelecimento, formas de reduzir a geração de resíduos e a reutilização de materiais, conhecimento das responsabilidades e tarefas, identificação das classes de resíduos, conhecimento sobre a utilização de veículos de coleta, uso de EPI e $E P C$, orientação sobre a biossegurança, higiene pessoal e ambiental, providências a serem tomadas em caso de determinados acidentes e situações emergenciais, visão básica do gerenciamento de resíduos sólidos do município e noções básicas do Controle de Infecção e de Contaminação Química". 
O laboratório E realiza um treinamento interno, onde o responsável por cada setor fica encarregado de ensinar as práticas para desenvolver o trabalho e acompanhar as atividades desempenhadas.

Por fim, os laboratórios foram questionados sobre os motivos que influenciam a adoção de práticas de gestão dos resíduos de serviços de saúde. No laboratório A segundo o gerente 1 "O laboratório sempre tentou andar em harmonia com a qualidade nos serviços prestados. O fato de ter implantado o PGRSS começou por ser uma exigência legal, mas não devemos cumprir a lei só por cumprir, cumpre prezando a qualidade, que se os resíduos pudessem ser descartados como a trinta anos atrás, seria mais simples e mais barato. Se as leis não evoluíssem e obrigassem a fazer de maneira correta, muitos não trabalhariam regularmente."

No laboratório B, os principais motivos são: para obter-se o descarte correto dos resíduos e evitar a contaminação do solo e das pessoas, tudo isso em função da lei. No laboratório $\mathrm{C}$, são adotados por dois fatores, primeiro, por questões da Biossegurança (do paciente, do colaborador e do transporte). Segundo, pela preocupação com o Meio Ambiente por se obter uma responsabilidade na gestão dos resíduos. Assim descreve o gestor 3 "É importante realizar um trabalho com uma consciência de que tenho que realizar um descarte final correto e ter consciência da minimização na geração de resíduos".

No laboratório $D$, as práticas de gestão são adotadas pela responsabilidade na gestão do descarte final dos resíduos e pelo cumprimento da legislação. E por fim, no laboratório E, são adotadas em primeiro plano, questões de obrigatoriedade em cumprimento da lei e a questão da preservação do meio ambiente vem em segundo plano. 


\section{CONSIDERAÇÕES FINAIS}

O setor da saúde tem passado por várias mudanças no que diz respeito à sua legislação, onde a busca pela garantia nos serviços prestados, e a veracidade dos laudos é fundamental. A procura pela qualidade de vida e a crescente demanda por serviços de saúde tem colaborado para o surgimento de diversos passivos, o que contribui para que os laboratórios se adequarem as novas mudanças exigidas pelo governo, onde o fator principal é a gestão dos resíduos por eles gerados. Deste modo, a Logística Reversa apresenta-se como uma ferramenta que tem contribuído positivamente para a sustentabilidade das práticas ambientais, cooperando para a realização do correto descarte final.

A pesquisa demonstrou que os laboratórios geram números elevados de resíduos no processo da prestação de seus serviços, e que se descartados de maneira inadequada, tais resíduos podem gerar uma diversidade de problemas. O presente estudo identificou que os laboratórios adotam práticas de gestão de descarte, em que realizadas algumas etapas de gerenciamento dos resíduos como: manejo, segregação, identificação, classificação, acondicionamento, armazenamento interno e externo e o descarte final dos resíduos por eles gerados, o que acontece por intermédio de uma empresa terceirizada contratada para a prestação do serviço.

Mediante os resultados apresentados por esta pesquisa, considera-se que os laboratórios de análises clínicas de Presidente Prudente/SP atendem às normas das Agências Reguladoras, tratando os seus resíduos conforme as normas exigidas na literatura, no entanto, esse comportamento está exclusivamente associado ao atendimento da legislação, tendo como fator secundário a segurança ambiental. 
